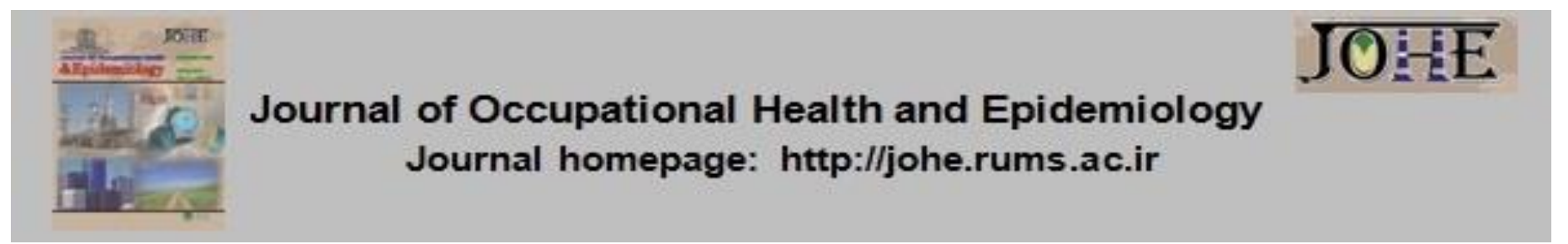

\title{
Psychological Consequences of the COVID-19 Disease among Physicians and Medical Students: A Survey in Kerman Province, Iran, in 2020
}

\author{
Sayed Mortaza Hossini Rafsanjanipoor ${ }^{1}$, Mohammad Ali Zakeri2², Mahlagha Dehghan ${ }^{3}$, Mahmood \\ Kahnooji ${ }^{4}$, Maryam Zakeri ${ }^{5}$ \\ 1. MSc in Epidemiology, Social Determinants of Health Research Centre, Rafsanjan University of Medical Sciences, Rafsanjan, Iran. \\ 2. MSc in Nursing, Social Determinants of Health Research Centre, Rafsanjan University of Medical Sciences, Rafsanjan, Iran. \\ 3. Assistant Prof., Dept. of Critical Care Nursing, Faculty of Nursing and Midwifery School, Kerman University of Medical Sciences, \\ Kerman, Iran. \\ 4. Assistant Prof., Dept. of Internal Medicine, Faculty of Medicine, Rafsanjan University of Medical Sciences, Rafsanjan, Iran. \\ 5. MSc in Physiology, Physiology-Pharmacology Research Center, Research Institute of Basic Medical Sciences, Rafsanjan \\ University of Medical Sciences, Rafsanjan, Iran.
}

Citation: Hossini Rafsanjanipoor SM, Zakeri MA, Dehghan M, Kahnooji M, Zakeri M.
Psychological Consequences of the COVID-19 Disease among Physicians and Medical
Students: A Survey in Kerman Province, Iran, in 2020. J Occu Health Epidemiol 2021;
10(4):274-81.

\section{Article Info}

* Corresponding author: Mohammad Ali Zakeri, E-mail: ma.zakeri115@gmail.com

\section{Article history}

Received: Aug 2021

Accepted: Dec 2021

10.52547/johe.10.4.274

Print ISSN: 2251-8096 Online ISSN: 2252-0902

Peer review under
responsibility of Journal of Occupational Health and Epidemiology

\begin{abstract}
Background: The Coronavirus (COVID-19) pandemic can have many adverse personal and professional consequences for physicians and medical students. Therefore, the present study aimed to investigate the psychological consequences of the COVID-19 disease in physicians and medical students during the pandemic.

Materials and Methods: Using the census method, this cross-sectional study was conducted on 132 physicians and medical students working in Rafsanjan hospitals, Kerman province, Iran, from May to August 2020. General Health Questionnaire (GHQ28), Generalized Anxiety Disorder 7-item (GAD-7), and Impact of Event Scale (IES) were used to collect data. The data were analyzed using SPSS 22, and descriptive and inferential statistics (Chi-square and Multiple logistic regression) methods were applied.

Results: More than half of the participants were female and married. According to GHQ, fifty-one and a half of the participants had psychological disorders. Based on the results, $24.2 \%$ of the participants had GAD, and $33.3 \%$ had PTSD. The results also showed that only occupation significantly correlated with psychological disorders $(P=0.01)$. The risk of psychological disorders in medical students was 2.38 times higher than among physicians $(\mathrm{OR}=2.38,95 \% \mathrm{Cl}=1.11-5.10, \mathrm{P}=0.026)$.

Conclusion: Physicians and medical students were faced too much stress in the COVID19 pandemic. They were at great risk of mental health disorders and the psychological consequences of COVID-19 due to their job condition. Managers and health policymakers need to take the necessary mental health interventions to reduce such risks.
\end{abstract}

Keywords: COVID-19, Health Status, Medical Students, Physicians.

\section{Introduction}

The COVID-19 pandemic is an international public health emergency unprecedented in modern history [1]. In addition to biological problems and the widespread and long-lasting changes in daily life, it may be difficult to adapt psychologically. Previous studies show that epidemics have social- psychological effects that eventually become more pervasive than the epidemic itself [2, 3]. Some social components, such as older adults, children, health care workers, infected patients, pre-existing mental patients, physicians, and medical students, are at higher risk of suffering from high levels of stress during the epidemic [4, 5]. Healthcare 
professionals are more likely to come in contact with carriers of the disease, putting them at greater risk of infection and spreading to others, including their relatives [6, 7]. At the same time, inexperience in such emergencies may be particularly stressful for physicians and medical students directly involved in the epidemic crisis [8]. The risk of the disease spreading and the recently reported infections in health care workers who have taken care of these patients can be the most important reasons for anxiety and stress in physicians and medical students [9]. Continuous spread of the disease, conspiracy theories, media reports on the disease, frustration/ boredom, lack of personal space in the home, and financial losses of the family are some of the important risk factors affecting the mental health of the two groups [10, 11]. In addition, the lack of personal protective equipment during a pandemic is a factor that increases the stress of physicians and medical students [12]. A review of previous epidemics, including the outbreak of Severe Acute Respiratory Syndrome (SARS) in 2003, shows that healthcare professionals can experience a wide range of psychological complications [13].

Medical staff disproportionately bear the extra physical and psychological burden associated with the disease; however, mental health outcomes are unknown in physicians and medical students [14, 15]. It has been reported that mental health problems among medical students are constantly increasing compared with students in other fields [16]. Mental health problems may continue into adulthood if left untreated [17]. These problems can have many adverse personal and professional consequences for physicians and medical students, including impaired quality of life, high risk of suicide, and reduced professional performance [18]. However, there are many unknowns about the psychological effects of this disease that need further research [19]. Therefore, it is necessary to gather information about the effects of the COVID19 pandemic on the mental health of specific groups, such as physicians and medical students, to help create appropriate interventions to reduce its adverse effects [20]. Regarding the prevalence of this disease in Iran and the need to study its psychological effects, this study is conducted to investigate the psychological consequences of the COVID-19 disease in physicians and medical students during its prevalence in a hospital in Rafsanjan county, Iran.

\section{Materials and Methods}

This cross-sectional study was conducted to assess the mental health of physicians and medical students in a hospital in Rafsanjan county, Kerman Province, Iran, during the pandemic of COVID-19 in 2020.

The research setting was Rafsanjan Ali-Ibn AbiTalib Hospital (in Kerman province, southeastern Iran) as the only referral hospital for Covid-19 patients in a great section of Kerman province in the southeastern area of the country. The statistical population included all physicians and medical students serving in this hospital. At present, about 150 physicians are working in this hospital, and more than 300 medical students are studying in different stages of general medicine and specialty courses.

In this study, all the statistical population was invited to participate. Inclusion criteria were fulltime attendance at the hospital for at least three months in the outbreak of COVID-19 disease and direct contact with patients. The exclusion criteria included failure to complete the questionnaire for any reason. Out of 160 questionnaires distributed among the eligible participants, 132 with a response rate of $82.5 \%$ were returned. Finally, data analysis was performed with 132 completed questionnaires.

Data were collected using four questionnaires, including socio-demographic form, General Health Questionnaire (GHQ -28), Generalized Anxiety Disorder 7-item (GAD-7), and Impact of Event Scale (IES).

Socio-demographic form: Participants' demographic information includes age, gender, marital status, occupation, job satisfaction, direct care for patients tested positive for coronavirus, relatives/ friends infected with the coronavirus, and the most important concern about the coronavirus. General Health Questionnaire (GHQ -28): The GHQ-28 was developed by Goldberg in 1972 to assess the general health of individuals and diagnose mental disorders [6]. It includes 28 items with four subscales of physical symptoms (7 items), anxiety symptoms and sleep disorders (7 items), social functioning (7 items), and depression symptoms ( 7 items). Each item is graded from 0 to 3. Therefore, the total scores of GHQ range from 0 to 84 , with higher scores reflecting greater psychological disorder. In addition, scores $\geq 23$ indicate people with psychological disorders [21]. In Rahmani et al.'s study, the reliability and validity of the questionnaire were confirmed, and the reliability was 0.85 using Cronbach's alpha in nurses working in the intensive care unit in the educational hospitals of Tabriz city [21]. In the present study, the Cronbach's alpha for GHQ was 0.93 .

Generalized Anxiety Disorder 7-item (GAD-7): The GAD-7 was developed by Spitzer et al. (2006) to 
measure worry and anxiety symptoms. The instrument has seven main items and one additional question to measure the degree of anxiety interference with a person's functioning. The questions are graded from 0 to 3 ("not at all," "several days," "more than half the days," and "nearly every day" scored as 0, 1, 2, and 3, respectively). Therefore, the total scores of GAD range from 0 to 21 , with higher scores reflecting greater anxiety. Scores $\geq 10$ are considered in the clinical range, i.e., the positive generalized anxiety disorder [22]. The GAD-7 has shown good reliability (confirmatory factor) and validity $(\alpha=$ 0.89 ) in the general population in Germany [23]. In Iran, the validity and reliability of this instrument were confirmed, and the Cronbach's alpha for the GAD-7 scale was 0.74 [24]. In the present study, Cronbach's alpha for the GAD-7 scale was 0.90 .

Impact of Event Scale (IES): Weiss and Marmar developed the IES in 1997 to measure psychological symptoms after a specific traumatic event. The scale has 22 items with three subscales of avoidance (8 items), intrusion (7 items), and hyperarousal ( 7 items). The questions are graded from 0 (not at all) to 4 (extremely). Therefore, the total scores of IES range from 0 to 88 , with higher scores reflecting greater post-traumatic stress disorder. Scores $\geq 33$ indicate people with PTSD [25]. The IES has shown good reliability and validity (alpha=0.96) in two samples of male Vietnam veterans [26]. In Iran, the validity and reliability of this instrument were confirmed, and the Cronbach's alpha for the IES was 0.87 among Iranian parents of children during cancer treatment [27]. In the present study, the Cronbach's alpha for the IES was 0.81 .

The researcher performed sampling after obtaining the necessary ethical permits from May to August 2020. Sampling was performed as a census by explaining the research objectives and obtaining informed consent from qualified individuals. The questionnaire was made available to physicians and medical students when they had enough free time to complete it.

SPSS 22 was used for data analysis. Frequency, percentage, as well as mean and standard deviation, were used to describe the sample characteristics, the GHQ, the GAD, and the PTSD scores. Participants with psychological disorders were identified according to each of the $G H Q$, GAD, and PTSD questionnaires. A Chi-squared test was used to check the association between qualitative variables and psychosocial disorders (yes/no). Multiple logistic regression was used to determine the association between significant variables and psychosocial disorders. All variables with a $p$-value $<0.2$ were included in the multiple logistic regression model. The significance level was considered 0.05 .

Ethics Committee of Rafsanjan University of Medical Sciences approved the study protocol (IR.RUMS.REC.1399.006). Information on the research objectives, information confidentiality/anonymity, and voluntary participation were explained to the participant on the first page of the questionnaire.

\section{Results}

The mean age of the participants was $33.21 \pm 8.77$ $($ Min $=23$ and Max $=53$ ). The mean age of the physicians was $39.83 \pm 8.31$, and the mean age of the students was $29.39 \pm 6.47$. Slightly more than half of the samples were female $(50.8 \%)$ and married $(50.8 \%, \mathrm{n}=67)$. Of 132 participants, $62.9 \%(\mathrm{n}=83)$ were students (interns and residents) and $37.1 \%(n=49)$ were physicians (general physicians and specialists). The majority of the participants were satisfied with their occupations $(63.8 \%, \mathrm{n}=81)$. Most of the participants who directly cared for patients were tested positive for coronavirus $(72.7 \%, \mathrm{n}=93)$. Nearly one-third of the participants had relatives/ friends infected with the coronavirus $(29.7 \%, \mathrm{n}=$ 38). Sixty-nine percent of the participants $(n=89)$ were most concerned about the infection of the family with the coronavirus (Table 1).

Table 1. Demographic characteristics of the physicians and medical students and their associations with psychological disorders in Rafsanjan County, Iran, in 2020 ( $N=132)$

\begin{tabular}{|c|c|c|c|c|c|}
\hline \multirow{2}{*}{\multicolumn{2}{|c|}{ Variables }} & \multicolumn{2}{|c|}{ Psychological disorders } & \multirow{2}{*}{ Total } & \multirow{2}{*}{$\begin{array}{c}\text { Chi-Square } \\
\text { test }\end{array}$} \\
\hline & & Yes $(n=78)$ & No $(n=54)$ & & \\
\hline \multirow{3}{*}{ Gender } & Male & $34(52.3)$ & $31(47.7)$ & $67(100)$ & $x 2=2.44$ \\
\hline & Female & $44(65.7)$ & $23(34.3)$ & $65(100)$ & $\mathrm{P}=0.12$ \\
\hline & Missing & $0(0.0)$ & $0(0.0)$ & $0(0.0)$ & \\
\hline \multirow{3}{*}{ Age (yr.) } & $\leq 30$ & $45(65.2)$ & $24(34.8)$ & $69(100)$ & $x^{2}=1.95$ \\
\hline & $>30$ & $33(53.2)$ & $29(46.8)$ & $62(100)$ & $\mathrm{P}=0.16$ \\
\hline & Missing & $0(0.0)$ & $1(100)$ & $1(100)$ & \\
\hline \multirow{3}{*}{ Marital status } & Married & $38(51.4)$ & $36(48.6)$ & $74(100)$ & $x^{2}=4.74$ \\
\hline & Unmarried & $40(70.2)$ & $17(29.8)$ & $57(100)$ & $P=0.03^{*}$ \\
\hline & Missing & $0(0.0)$ & $1(100)$ & $1(100)$ & \\
\hline
\end{tabular}




\begin{tabular}{|c|c|c|c|c|c|}
\hline \multirow{3}{*}{ Occupation } & Student & $56(67.5)$ & $27(32.5)$ & $83(62.9)$ & $x^{2}=6.49$ \\
\hline & Physician & $22(44.9)$ & $27(55.1)$ & 49 (37.1) & $P=0.01^{*}$ \\
\hline & Missing & $0(0.0)$ & $0(0.0)$ & $0(0.0)$ & \\
\hline \multirow{3}{*}{ Job satisfaction } & Yes & $44(54.3)$ & $37(45.7)$ & $81(63.8)$ & \multirow{3}{*}{$\begin{array}{l}X 2=2.84 \\
P=0.09^{*}\end{array}$} \\
\hline & No & $32(69.6)$ & $14(30.4)$ & $46(36.2)$ & \\
\hline & Missing & $2(40.0)$ & $3(60.0)$ & $5(100)$ & \\
\hline \multirow{3}{*}{$\begin{array}{l}\text { Caring directly for } \\
\text { patients tested positive } \\
\text { for coronavirus }^{\mathrm{a}}\end{array}$} & Yes & $52(55.9)$ & $41(44.1)$ & $93(72.7)$ & \multirow{3}{*}{$\begin{array}{l}2=1.69 \\
P=0.19^{*}\end{array}$} \\
\hline & No & $24(68.6)$ & $11(31.4)$ & $35(27.3)$ & \\
\hline & Missing & $2(50.0)$ & $2(50.0)$ & $4(100)$ & \\
\hline \multirow{3}{*}{$\begin{array}{l}\text { Relatives/ friends } \\
\text { infected with the } \\
\text { coronavirus }\end{array}$} & Yes & $18(47.4)$ & $20(52.6)$ & $38(29.7)$ & \multirow{3}{*}{$\begin{array}{l}X 2=2.81 \\
P=0.09\end{array}$} \\
\hline & No & $57(63.3)$ & $33(36.7)$ & $90(70.3)$ & \\
\hline & Missing & $3(75.0)$ & $1(25.0)$ & $4(100)$ & \\
\hline \multirow{4}{*}{$\begin{array}{l}\text { The most important } \\
\text { concern about the } \\
\text { coronavirus }\end{array}$} & My family getting sick & $52(58.4)$ & $37(41.6)$ & $89(69.0)$ & \multirow{4}{*}{$\begin{array}{l}X 2=0.03 \\
P=0.99\end{array}$} \\
\hline & Death & $18(58.1)$ & $13(41.9)$ & $31(24.0)$ & \\
\hline & Others & $5(55.6)$ & $4(44.4)$ & $9(7.0)$ & \\
\hline & Missing & $3(100.0)$ & $0(0.00)$ & $3(100)$ & \\
\hline
\end{tabular}

The mean score of general health was $25.28 \pm$ 12.71. Of 132 participants, 68 (51.5\%) had psychological disorders. The mean score of the general health of the physicians and students was $20.81 \pm 10.56$ and $27.91 \pm 13.18$, respectively. Among the GHQ subscales, social functioning had the highest score, and depression had the lowest score. On the other hand, $73.5 \%(n=97)$ of the participants had social functioning disorder, $48.5 \%$ ( $n=64)$ had physical symptoms, $50.8 \%(n=67)$ had anxiety symptoms and sleep disorders, and $19.7 \%(n=26)$ had depression. In addition, $24.2 \%$ $(\mathrm{n}=32$ ) of the participants had GAD and $33.3 \%$ ( $n$ $=44)$ had PTSD (Table 2). Totally, 40.9\% $(n=54)$ of the participants had no psychological disorders, $23.5 \%(n=31)$ had one psychological disorder, $21.2 \%(n=28)$ had two psychological disorders, and $14.4 \%(n=19)$ had all three psychological disorders.

Table 2. The physicians and medical students' general health condition, generalized anxiety, and post-traumatic stress disorder in Rafsanjan County, Iran, in 2020 ( $N=132$ )

\begin{tabular}{ccccc}
\hline Variable & Minimum & Maximum & Mean \pm SD & $\begin{array}{c}\text { People with a disorder } \\
(\mathbf{N} / \%)\end{array}$ \\
\hline General health & 4 & 56 & $25.28 \pm 12.71$ & $68(51.5)$ \\
\hline Physical symptoms & 0 & 17 & $6.94 \pm 3.88$ & $64(48.5)$ \\
\hline Anxiety symptoms and sleep disorders & 0 & 20 & $6.76 \pm 4.66$ & $67(50.8)$ \\
\hline Social functioning & 0 & 20 & $8.21 \pm 3.18$ & $97(73.5)$ \\
\hline Symptoms of depression & 0 & 20 & $3.37 \pm 4.17$ & $26(19.7)$ \\
\hline Generalized anxiety disorder & 0 & 20 & $6.51 \pm 5.01$ & $32(24.2)$ \\
\hline Post-traumatic stress disorder & 0 & 54 & $27.0 \pm 12.68$ & $44(33.3)$ \\
\hline
\end{tabular}

The bivariate analysis (Chi-squared test) showed a significant association between psychological disorders with marital status and occupation (Table 1). For further analysis, the multiple logistic regression with the backward method was conducted. All variables with a p-value $<0.2$ included in the multiple logistic regression model (i.e., gender, age, marital status, occupation, job satisfaction, caring directly for patients tested positive for coronavirus, relatives/ friends infected with the coronavirus). The results showed that only occupation significantly correlated with psychological disorders. On the other hand, the risk of psychological disorders in medical students was 2.38 times higher than physicians $(95 \%$ Confidence Interval for odds ratio: 1.11- 5.10, P = 0.026) (Table 3).

Table 3. The logistic model of the associations between important variables and psychological disorders in the physicians and medical students in Rafsanjan County, Iran, in 2020 ( $N=132)$

\begin{tabular}{ccccc}
\hline \multirow{2}{*}{ Variable* } & \multicolumn{3}{c}{ Multiple logistic regression } \\
\cline { 2 - 5 } & Odds ratio & 95\% Confidence interval for odds ratio & P-value \\
\hline \multirow{2}{*}{ Occupation } & Physician & 1 & $1.11-5.10$ & 0.026 \\
\cline { 2 - 5 } & Student & 2.38 & 10 & \\
\hline
\end{tabular}

$1=$ the reference

* Sex, age, marital status, job satisfaction, caring directly for patients tested positive for coronavirus, and relatives/ friends infected with the coronavirus also were included in the logistic model with the backward method; however, none of them remained in the final model. 


\section{Discussion}

The outbreak of the COVID-19 pandemic has caused a global crisis of general and mental health, as well as a huge psychosocial experience [28]. This study examined the psychological consequences (including general health, general anxiety disorder, and post-traumatic stress disorder) of the COVID-19 disease in physicians and medical students during its outbreak. The results reflected the psychological effects of the COVID-19 pandemic on the mental health of the who groups in Iran.

Based on the results, the scores of almost half of physicians and medical students participating in this study were higher than the threshold of the $\mathrm{GHQ}$, indicating significant mental health problems. In addition, the highest score was related to the dimension of social functioning, and the lowest score was related to the dimension of depression, so that $73.5 \%$ of the participants had social dysfunction. These results have been confirmed in studies on the prevalence of the disease in Iranian society [29-31] and other communities [10, 11]. Disturbance of individual structures reduces control of the individual and the predictability of the flow of life [32]. Hence, social support reduces anxiety and stress and improves self-efficacy [33]. Socioemotional support and empathy of friends or family members can help medical staff reduce anxiety [34]. Social support can help reduce stress by reducing the perception of threat from stressful events, resulting in the right physiological response [33].

Twenty-four-tenths of a percent of the participants in our study had symptoms of anxiety, which was higher than that in the studies of Rabih $\mathrm{H}$ et al. (United Arab Emirates) (19.8\%) [4] and Wei Deng et al. (21\%) [9] that were performed on the general population and healthcare workers. However, the anxiety symptom percentage in our study was lower than that in the study of Chang Jinghui [35] ( $28.9 \%$ mild, $11.5 \%$ moderate, and $7.4 \%$ severe). Numerous studies have examined the psychological implications of the COVID-19 pandemic on the mental health of students and physicians, particularly in China. Chang Jinghui \& et al. (2020) showed that $26.6 \%$ of the medical students in Guangdong Province, China, had anxiety symptoms, and $21.16 \%$ of them had symptoms of depression [35]. Furthermore, Wei Deng in China (2019) found that the prevalence of depression in medical students was 29\% [9]. Naseem Ahmed (2020) examined the concerns of medical students about the prevalence of COVID19 and showed that $75.8 \%$ of participants were worried about the possibility of developing the disease and $80 \%$ of them were afraid of inadequate treatment and care [36].

Rabih Halvani in the United Arab Emirates showed that approximately half of the medical students reported varying degrees of anxiety, from mild to severe, during hospital visits [4]. Muhammad Salman et al. in Pakistan found that COVID-19 had a significant adverse impact on students' mental health [37].

Among the few studies on the psychological consequences of the COVID-19 outbreak among physicians, Civantos AM showed that $47.9 \%$ of the participants $(\mathrm{N}=349)$ were anxious $(28.9 \%$ mild, $11.5 \%$ moderate, $7.4 \%$ severe), and $21.8 \%$ were depressed. Furthermore, $60.2 \%$ of the physicians reported symptoms of distress (EIS) $(32.7 \%$ mild, $20.9 \%$ moderate, and $6.6 \%$ severe) [38]. Another study in China found that $11.4 \%$ and $45.6 \%$ of the physicians had symptoms of anxiety and depression, respectively [39]. A study in Iran found that $68.5 \%$ of the physicians caring for COVID-19 patients suffered from anxiety [40]. Anxiety is the most fundamental characteristic of critical situations, and the unpredictability of the future has the greatest role in creating it [41]. Therefore, it can negatively affect people's mental health. This study showed that the COVID-19 pandemic had negative adverse effects on the health of psychiatrists and medical students in Iran.

The recent COVID-19 pandemic has features such as an unprecedented number of patients with severe and often unpredictable symptoms, high mortality, and lack of effective treatments that increase the risk of PTSD among medical students. PTSD is a severe mental illness that may develop in individuals who have experienced or witnessed a traumatic event [42]. Hence, the recent outbreak burden on medical students and physicians deserves much more attention because they are more likely to be involved in diagnosing and treating COVID-19 patients and more prone to mental disorders, including PTSD.

Thirty-three and three-tenths of a percent of the participants in our study had PTSD symptoms that were higher than that in the studies of Sen Chen in China [43] and Apostolos Blekas in Greece (43), which were performed on undergraduate student and healthcare workers. Such a discrepancy may be because the participants in this study had different levels of contact with the COVID-19 patients, possibly increasing the fear of transmitting the disease. Fear is an adaptive response to defensive behaviors to protect oneself from danger, followed by PTSD when improperly adjusted [44]. Regarding the contagious nature of the COVID-19 and its long incubation period (14 days or more), many participants may be afraid of 
inadvertent contact with the disease and spreading it to other family members. Therefore, continuing education for better understanding the disease and decreasing the risks of COVID-19 disease can play an effective role in reducing the impact [45]. Also, it is helpful to perform psychological interventions to reduce fear of pandemics, induce psychological resilience, and prevent PTSD.

According to the results of this study, although there was no statistically significant difference between mental health disorder, age, and sex, the mental health disorder of women was higher than that of men, indicating that women usually have a higher rate of psychological distress than men. Because female students have less social support, they may suffer from a decreased sense of cohesion, which in turn is a strong explanatory variable for psychological distress in medical students in general and female students in particular [46]. In addition, the higher prevalence of mental disorders in female students than those of males can be due to biological factors, the role of gender, environmental stress, poor satisfaction, and limited social participation of girls in society. Other reasons include stress management methods in both sexes [47]. The results showed that among the study variables, only the job was associated with psychological disorders. On the other hand, the risk of psychological disorders in medical students was 2.38 times higher than that of the physicians, which may be due to the greater work experience of the latter group in dealing with such situations.

One of the limitations of this study was the limited studies on the prevalence of depression, anxiety, and mental health disorders among medical students and physicians during the COVID-19 epidemic worldwide, and in particular, in the Middle East; thus, it was not possible to compare our findings with similar environments and cultures. In addition, the cross-sectional nature of this study did not allow us to determine the cause and effect relationship between psychological complications and stress. It may be helpful to repeat the study after the peak of the epidemic to determine the effect of time on results. Despite these limitations, the present study appears to be unique, which has used a standardized measure to quantify the mental health of medical students.

However, further studies on larger sample sizes are recommended to describe the causes, consequences, and solutions to these problems. Repeating these studies at other academic centers and conducting similar studies are other suggestions.

\section{Conclusion}

Physicians and medical students are under much psychological stress during the outbreak of COVID-19, thus being at greater risk for mental health disorders, social dysfunction, anxiety, and depression. Therefore, the psychological state, especially among medical students, should be improved more carefully to eliminate risk factors and prevent the exacerbation of these disorders. The results of this study can be a guide to activate student-counseling centers and study their problems. It seems necessary to take measures to identify and eliminate the factors influencing the occurrence of mental disorders.

\section{Acknowledgement}

This study is part of research project No. IR.RUMS.98311. We would thank the authorities of the Social Determinants of Health Research Centre, Rafsanjan University of Medical Sciences, students, and physicians participating in this project.

Conflict of interest: None declared.

\section{References}

1. Moradi-Joo M, Babazadeh T, Honarvar Z, Mohabat-Bahar S, Rahmati-Najarkolaei F, Haghighi M. The Relationship between Spiritual Health and Public Health Aspects among Patients with Breast Cancer. Res Relig Health 2017; 3(3):80-91.

2. Ornell F, Schuch JB, Sordi AO, Kessler FHP. "Pandemic fear" and COVID-19: mental health burden and strategies. Braz J Psychiatry 2020; 42(3):232-5.

3. Li Z, Ge J, Yang M, Feng J, Qiao M, Jiang R, et al. Vicarious traumatization in the general public, members, and non-members of medical teams aiding in COVID-19 control. Brain Behav Immun 2020; 88:916-9.

4. Saddik B, Hussein A, Sharif-Askari FS, Kheder W, Temsah $\mathrm{MH}$, Koutaich RA, et al. Increased Levels of Anxiety among Medical and NonMedical University Students during the COVID19 Pandemic in the United Arab Emirates. Risk Manag Healthc Policy 2020; 13:2395.

5. Zakeri MA, Hossini Rafsanjanipoor SM, Sedri N, Kahnooji M, Sanji Rafsanjani M, Zakeri M, et al. Psychosocial status during the prevalence of COVID-19 disease: the comparison between healthcare workers and general population. Curr Psychol 2021:1-9.doi:10.1007/s12144-02101582-1.

6. Zakeri MA, Hossini Rafsanjanipoor SM, Zakeri 
M, Dehghan M. The relationship between frontline nurses' psychosocial status, satisfaction with life and resilience during the prevalence of COVID-19 disease. Nurs Open 2021; 8(4):1829-39.

7. Zakeri MA, Rahiminezhad E, Salehi F, Ganjeh $\mathrm{H}$, Dehghan M. Burnout, Anxiety, Stress, and Depression among Iranian Nurses: Before and During the First Wave of the COVID-19 Pandemic. Front Psychol 2021; 12:789737.

8. Gao J, Zheng P, Jia Y, Chen H, Mao Y, Chen S, et al. Mental health problems and social media exposure during COVID-19 outbreak. PLPoS One 2020; 15(4):e0231924.

9. Zeng W, Chen R, Wang X, Zhang Q, Deng W. Prevalence of mental health problems among medical students in China: A meta-analysis. Medicine (Baltomore) 2019; 98(18):e15337.

10. Wang C, Pan R, Wan X, Tan Y, Xu L, Ho CS, et al. Immediate Psychological Responses and Associated Factors during the Initial Stage of the 2019 Coronavirus Disease (COVID-19) Epidemic among the General Population in China. Int J Environ Res Public Health 2020; 17(5):1729.

11. Cao W, Fang Z, Hou G, Han M, Xu X, Dong J, et al. The psychological impact of the COVID-19 epidemic on college students in China. Psychiatry Res 2020; 287:112934.

12. Devnani M. Factors associated with the willingness of health care personnel to work during an influenza public health emergency: an integrative review. Prehosp Disaster Med 2012; 27(6):551-66.

13. Styra R, Hawryluck L, Robinson S, Kasapinovic S, Fones C, Gold WL. Impact on health care workers employed in high-risk areas during the Toronto SARS outbreak. J Psychosom Res 2008; 64(2):177-83.

14. Lai J, Ma S, Wang Y, Cai Z, Hu J, Wei N, et al. Factors Associated with Mental Health Outcomes among Health Care Workers Exposed to Coronavirus Disease 2019. JAMA Netw Open 2020; 3(3):e203976.

15. Goulia P, Mantas C, Dimitroula D, Mantis D, Hyphantis T. General hospital staff worries, perceived sufficiency of information and associated psychological distress during the $\mathrm{A} / \mathrm{H} 1 \mathrm{~N} 1$ influenza pandemic. BMC Infect Dis 2010; 10:322.

16. Stewart-Brown S, Evans J, Patterson J, Petersen S, Doll H, Balding J, et al. The health of students in institutes of higher education: an important and neglected public health problem? J Public Health Med 2000; 22(4):492-9.

17. Pine DS, Cohen P, Gurley D, Brook J, Ma Y. The risk for early-adulthood anxiety and depressive disorders in adolescents with anxiety and depressive disorders. Arch Gen Psychiatry 1998; 55(1):56-64.

18. Lins L, Carvalho FM, Menezes MS, Porto-Silva $\mathrm{L}$, Damasceno H. Health-related quality of life of students from a private medical school in Brazil. Int J Med Educ 2015; 6:149-54.

19. Huang C, Wang Y, Li X, Ren L, Zhao J, Hu Y, et al. Clinical features of patients infected with 2019 novel coronavirus in Wuhan, China. Lancet 2020; 395(10223):497-506.

20. Löwe B, Decker O, Müller S, Brähler E, Schellberg D, Herzog W, et al. Validation and standardization of the Generalized Anxiety Disorder Screener (GAD-7) in the general population. Med Care 2008; 46(3):266-74.

21. Rahmani F, Behshid M, Zamanzadeh V, Rahmani F. Relationship between general health, occupational stress and burnout in critical care nurses of Tabriz teaching hospitals. Iran J Nurs 2010; 23(66):54-63.

22. Modanloo M. The challenge of increasing adherence in schizophrenia: Focusing future research on changing attitudes of patients. Asian J Psychiatr 2018; 36:28.

23. Löwe B, Decker O, Müller S, Brähler $E$, Schellberg D, Herzog W, et al. Validation and standardization of the Generalized Anxiety Disorder Screener (GAD-7) in the general population. Med Care 2008; 46(3):266-74.

24. Naeinian MR, Shaeiri MR, Sharif M, Hadian M. To Study Reliability and Validity for A Brief Measure for Assessing Generalized Anxiety Disorder (GAD-7). Clin Psychol Personal 2011; 9(1):41-50.

25. Weiss DS, Marmar CR. The Impact of Event Scale-Revised. In: Wilson JP, Keane TM, Eds. Assessing psychological trauma and PTSD. 1st ed. New York, NY, United States: Guilford Press; 1996.

26. Creamer M, Bell R, Failla S. Psychometric Properties of the Impact of Event ScaleRevised. Behav Res Ther 2003; 41(12):148996.

27. Iranmanesh S, Shamsi A, Dehghan M. PostTraumatic Stress Symptoms among Iranian Parents of Children during Cancer Treatment. Issues Ment Health Nurs 2015; 36(4):279-85.

28. Zakeri MA, Hossini Rafsanjanipoor SM, Kahnooji M, Ghaedi Heidari F, Dehghan M. Generalized Anxiety Disorder During the COVID-19 Outbreak in Iran: The Role of Social Dysfunction. J Nerv Ment Dis 2021; 209(7):4916.

29. Hossini Rafsanjanipoor SM, Zakeri MA, Dehghan M, Kahnooji M, Sanji Rafsanjani M, Ahmadinia $\mathrm{H}$, et al. Iranian psychosocial status and its determinant factors during the prevalence of COVID-19 disease. Psychol Health Med 2021:1-12. doi:10.1080/13548506.2021.1874438.

30. Zakeri MA, Dehghan M, Ghaedi Heidari F, Pakdaman H, Mehdizadeh M, Ganjeh H, et al. Mental health outcomes among health-care workers during the COVID-19 outbreak in Iran. Ment Health Rev J 2021; 26(2):152-60.

31. Zakeri MA, Maazallahi M, Ehsani V, Dehghan 
M. Iranian Psychosocial status during and after COVID-19 outbreak mandatory quarantine: A cross-sectional study. J Community Psychol 2021; 49(7):2506-16.

32. Rubin GJ, Wessely S. The psychological effects of quarantining a city. BMJ 2020; 368:m313.

33. Adamczyk K, Segrin C. Perceived Social Support and Mental Health among Single vs. Partnered Polish Young Adults. Curr Psychol 2015; 34(1):82-96.

34. Yang $\mathrm{N}$, Xiao $\mathrm{H}$, Wang W, Li S, Yan H, Wang Y. Effects of doctors' empathy abilities on the cellular immunity of patients with advanced prostate cancer treated by orchiectomy: the mediating role of patients' stigma, self-efficacy, and anxiety. Patient Prefer Adherence 2018; 12:1305-14.

35. Chang J, Yuan Y, Wang D. [Mental health status and its influencing factors among college students during the epidemic of COVID-19]. Nan Fang Yi Ke Da Xue Xue Bao 2020; 40(2):171-6.

36. Ahmed N, Khan A, Naveed HA, Moizuddin SM, Khan J. Concerns of Undergraduate Medical Students Towards an Outbreak of Covid-19. Int J Curr Med Pharm Res 2020; 6(3):5055-62.

37. Salman M, Asif N, Mustafa ZU, Khan TM, Shehzadi N, Hussain K, et al. Psychological Impact of COVID-19 on Pakistani University Students and How They Are Coping. Medrxiv [Internet]. 2020 Jan 1. Available from: https://doi.org/10.1101/2020.05.21.20108647

38. Civantos AM, Byrnes Y, Chang C, Prasad A, Chorath K, Poonia SK, et al. Mental health among otolaryngology resident and attending physicians during the COVID-19 pandemic: National study. Head Neck 2020; 42(7):15971609.

39. Zhu J, Sun L, Zhang L, Wang H, Fan A, Yang $B$, et al. Prevalence and Influencing Factors of Anxiety and Depression Symptoms in the First-
Line Medical Staff Fighting Against COVID-19 in Gansu. Front Psychiatry 2020; 11:386.

40. Hassannia L, Taghizadeh F, Moosazadeh M, Zarghami M, Taghizadeh $\mathrm{H}$, Dooki AF, et al. Anxiety and Depression in Health Workers and General Population During COVID-19 in IRAN: A Cross-Sectional Study. Neuropsychopharmacol Rep 2021; 41(1):40-9.

41. Grupe DW, Nitschke JB. Uncertainty and anticipation in anxiety: an integrated neurobiological and psychological perspective. Nat Rev Neurosci 2013; 14(7):488-501.

42. Sareen J. Posttraumatic stress disorder in adults: impact, comorbidity, risk factors, and treatment. Can J Psychiatry 2014; 59(9):460-7.

43. Tang W, Hu T, Hu B, Jin C, Wang G, Xie C, et al. Prevalence and correlates of PTSD and depressive symptoms one month after the outbreak of the COVID-19 epidemic in a sample of home-quarantined Chinese university students. J Affect Disord 2020; 274;1-7.

44. Malakoutikhah A, Zakeri MA, Salehi Derakhtanjani A, Dehghan M. Anxiety, anger, and mindfulness as predictors of general health in the general population during COVID-19 outbreak: A survey in southeast Iran. J Community Psychol 2021. doi:10.1002/jcop.22690.

45. Zakeri MA, Dehghan M. The Role of Continuing Education in Protecting Nurses against COVID19 Infection. J Occu Health Epidemiol 2021; 10(2):64-6

46. Bíró É, Balajti I, Ádány R, Kósa K. Determinants of mental well-being in medical students. Soc Psychiatry Psychiatr Epidemiol 2010; 45(2):2538.

47. Debiec KE, Paul KJ, Mitchell CM, Hitti JE. Inadequate prenatal care and risk of preterm delivery among adolescents: a retrospective study over 10 years. Am J Obstet Gynecol 2010; 203(2):122.e1-6. 